\title{
An immunohistochemical study of CD83- and CD1a-positive dendritic cells in the decidua of women with recurrent spontaneous abortion
}

\author{
Zhi-Da Qian ${ }^{1}$, Li-Li Huang ${ }^{1}$ and Xiao-Ming Zhu ${ }^{1,2^{*}}$
}

\begin{abstract}
Background: There are more and more women with recurrent spontaneous abortion (RSA). The mechanism of RSA is still unclear. Immunological factors have been postulated to play a role in the etiology of RSA. Dendritic cells (DCs) are the most potent antigen-presenting cells in the immune system, and the decidual DCs may take part in the occurrence of RSA. The difference in maturity status of decidual DCs among women with RSA and women with normal pregnancies is worthy of studying for its application to prevention and therapy.

Methods: The EnVision two-step immunohistochemical staining technique was used to detect the expression of CD83 and CD1a in the decidua of women with RSA (30 cases) and normal pregnancies (30 cases). The maturity status, distribution and quantity of DCs in the two groups were observed. Observation of the staining and cell counting were done using microscope within 30 randomly selected high-power fields (HPF, $40 \times 10)$. All data analyses were conducted with SPSS 17.0 and the statistical significance was set at $P<0.05$.
\end{abstract}

Results: The decidua from the two groups contained DCs that stained with the anti-CD83 and anti-CD1a antibody. Most of the decidual $C D 83^{+} D C$ s from two groups were located in the stroma. There were more $C D 83^{+} D C s$ clustered with other DCs in the stroma from women with RSA than normal pregnancies. Most of the CD1a ${ }^{+} D C s$ in the decidua from the two groups are located close to maternal glandular epithelium. No difference in the location of $C D 1 a^{+} D C s$ was found in the decidua between two groups. The number of decidual CD $83^{+}$DCs was statistically significantly higher in RSA women than in normal early pregnant women (14.20 $\pm 13.34 / 30 \mathrm{HPF}$ versus 4.77 $\pm 2.64 / 30$ HPF; $t=3.800, P=0.001)$. The number of CD1a ${ }^{+} D C s$ in the decidua was statistically significantly lower in RSA women compared with normal early pregnant women (3.97 $\pm 3.75 / 30 \mathrm{HPF}$ versus $7.60 \pm 6.08 / 30 \mathrm{HPF} ; t=2.786, P=0.008)$.

Conclusions: These findings suggest that the increase in the number of mature DCs and the decrease in the quantity of immature DCs in the decidua may be related to RSA. The maturation of decidual DCs may play an important role in the pathogenesis of RSA.

Keywords: recurrent spontaneous abortion, decidua, dendritic cells, CD83, CD1a, immunohistochemistry

\section{Background}

Miscarriage is the most common complication of pregnancy. Recurrent spontaneous abortion (RSA) is defined as the miscarriage of three or more consecutive pregnancies before 20 weeks of gestation and affects about $1 \%$ of infertile couples [1]. Pregnancy is a phenomenon of

\footnotetext{
* Correspondence: fbzhuxiaoming@163.com

'Department of Obstetrics and Gynecology, Women's Hospital, School of Medicine, Zhejiang University, 1 Xueshi Road, Hangzhou, Zhejiang Province 310006, People's Republic of China

${ }^{2}$ Key Laboratory of Reproductive Genetics (Zhejiang University), Ministry of Education, Hangzhou, People's Republic of China
}

successful semi-allograft. Its success depends on the mother's immune tolerance of pregnancy. Pathological pregnancy may occur if this tolerance is damaged. The incidence of RSA is influenced by a number of pregnancyrelated factors, including anatomic, infectious, endocrine, environmental, genetic and immunologic factors. Immunological factors have been postulated to play a role in the etiology of recurrent miscarriage as the fetus and placenta is semi-allogenic to the mother. Study in the $C B A / J \times D B A /$ $2 J$ mouse model showed that the use of syngeneic DC prevented abortions [2]. There has been a growing interest 
in the study of immunological factors of RSA. Maternal and fetal immune cells come into direct contact in the decidua, which plays a key role in fetal tolerance. Abnormal immune tolerance of maternal-fetal interface of RSA is related to the dysregulation of human leukocyte antigen (HLA) and apoptosis of natural killer (NK) cells, T lymphocytes, macrophages, dendritic cells (DCs), and other immune cells $[3,4]$.

DCs are the most potent antigen-presenting cells (APC) in the immune system with the unique ability to induce primary immune responses [5]. DCs play an important role in the initiation and regulation of immune responses by regulating $\mathrm{T}$ cell-mediated immunity $[6,7]$. DCs also play an important role in inducing immune tolerance [8]. DCs are derived from bone marrow stem cells, migrate through the blood, and then disperse widely in lymphoid tissues and nonlymphoid tissues, such as liver, heart, kidney and lung tissue (except brain). There are two entities of DCs that differ phenotypically and functionally, the mature and immature DCs (mDCs and iDCs) $[7,9]$. The iDCs are excellent at antigen uptake, but are poor antigen presenters, and the reverse is true for the mature subgroup. The iDCs transform into $\mathrm{mDCs}$ and induce immune response under the influence of maturesignals $[7,10]$. The differing character in the two groups of DCs is the accessory molecule expression that can be studied immunohistochemically. CD83 is a marker of mDCs [11], and CD1a is a marker of iDCs [12].

DCs exist in normal endometrium and pregnancy decidua $[10,13]$. Uterine DCs in the decidua have been implicated in pregnancy maintenance. In early pregnancy, PGE2 and IL-10 in the decidua can lead to the generation of tolerant DCs $[14,15]$. The level of IL-10 in placental tissue gradually increased with the development of normal pregnancy, and high concentrations of IL-10 may inhibit the ability of DCs to produce IL-12, and the balance of the T-helper-1 type response/T-helper-2 type response (Th1/Th2) is shifted to the Th2 direction. The iDCs in the decidua of a normal pregnancy do not express CD83 molecules [16]. This suggests that the formation of maternal-fetal immune tolerance may be related to the immature status of DCs in the microenvironment of the maternal-fetal interface. Blois et al. [2] studied the effect of adoptive transfer of DCs on the maintenance of pregnancy in the $C B A / J \times D B A / 2 J$ mouse model. They found that the control (no treatment) abortion rate was $23.8 \%$, and with GM-CSF alone was $17.6 \%$. The abortion rate was reduced to $2.2 \%$ after inoculation of syngeneic DCs. It suggested that syngeneic DCs may have a significant protective effect in miscarriage in pregnant mice. DCs may not only have mediated the defensive immune response, but also the tolerance of embryos. There was a fine balance in the interaction between DCs and trophoblast cells during successful pregnancy in mice. DCs therapy could upregulate a regulatory/protective population of cells at the maternal-fetal interface $[17,18]$. Askelund et al. [19] found there were significantly more CD $83^{+}$DCs in the decidua from women with RSA than normal pregnancies at 8 weeks' gestation. It suggested that DCs may be associated with any immune-related pathological pregnancy, and DCs may play an important role in the pathogenesis of RSA. However, little is known about the underlying mechanisms. The difference in maturity status of decidual DCs between women with RSA and normal pregnancies is worthy of studying for its application to prevention and therapy. This study examines the mature and immature DC density using CD83 and CD1a antibody, respectively.

\section{Methods}

\section{Patients}

This is a prospective study of DCs in the uterine decidua. The specimens were obtained from the Department of Obstetrics and Gynecology, Women's Hospital, School of Medicine, Zhejiang University, China. Samples of RSA group (30 cases) were collected from women with RSA by ultrasound guided curettage immediately upon confirmation of embryonic demise. Time delay between embryonic demise and curettage was no more than 2 days. Samples of the control group (30 cases) were collected from women who underwent elective termination of normal pregnancies without history of miscarriages in the same hospital. All women were from 20 to 40 years of age with gestation of less than 14 weeks. There was no significant difference between the general information of the two groups (Table 1). Excluded from the study were women with genetic, endocrine and immune disorders, cancer, genital anatomical abnormalities and major surgical diseases, history of long-term medication, trophoblastic disease and those undergoing endocrine therapy, chemotherapy, and radiotherapy. The products of conception and the parents were karyotyped to exclude chromosomal abnormalities in the RSA group. Written informed consent was obtained from each patient and the study was approved by the Institutional Review Board of Women's Hospital, School of Medicine, Zhejiang University, China.

\section{Immunohistochemistry}

Decidua were immediately placed into a $10 \%$ formaldehyde solution bag and fixed for 10 to 24 hours. Paraffin blocks were sectioned into a 4- $\mu \mathrm{m}$ thickness. The EnVision two-step immunohistochemical staining technique was used to detect the expression of CD83 and CD1a in the decidua. The immunohistochemical staining was done by one person only. Positive controls were included in every batch of tests. Primary antibodies: Mouse antihuman 
Table $1 \mathrm{General}$ information for the two groups

\begin{tabular}{lllll}
\hline & N & Age (years; mean \pm SD) & Gestational age (days; mean \pm SD) & Abortions (mean \pm SD) \\
\hline RSA & 30 & $29.77 \pm 4.45$ & $64.17 \pm 11.36$ & $4.33 \pm 1.52$ \\
Normal pregnancy & 30 & $28.87 \pm 5.84$ & $59.03 \pm 9.35$ & $3.93 \pm 0.52$ \\
$P$ & & 0.504 & 0.073 & 0.180 \\
\hline
\end{tabular}

There was no significant difference between the general information for the two groups. RSA, recurrent spontaneous abortion.

CD83 (Model NCL-CD83, clone number 1H4b) was obtained from Novocastra Biotechnology Co., Ltd, US. Mouse antihuman CD1a (Model GM357104, clone number 010) was obtained from DAKO Biotechnology Co., Ltd, Denmark. The secondary antibody used was ChemMatei Envisioni Detection Kit Peroxidase/DAB, from DAKO Biotechnology Co., Ltd, Denmark. The normal tonsil was used as a CD83 positive control, and the normal skin was used as a CD1a positive control.

\section{Dendritic cell counts}

DCs displayed membrane staining, cytoplasmic staining, and branch-like morphological features. MDCs were characterized by the expression of CD83, and iDCs were characterized by the expression of CD1a. The location and number of DCs in the two groups were observed within 30 randomly selected high-power fields (HPF, $\times 40$ objective and $\times 10$ eye piece). Observation of the staining and cell counting were done using a Olympus CHK microscope (Olympus, Japan). Photomicrographs were taken using a digital camera (Nikon, Japan). All the immunostained slides were reviewed by two observers independently.

\section{Statistics}

The difference in the counts of DCs between the two groups was evaluated. All data analyses were conducted with SPSS 17.0 (SPSS Inc., USA). Statistical significance was set at $P<0.05$, and $P$ values from all tests were reported. The statistical significance of the experimental differences in the two groups was assessed by a normal distribution test first. If the data were normally distributed, an independent samples $t$ test for continuous variables analysis was used, and if the data were not normally distributed, a non-parametric test (Mann-Whitney Test) was used.

\section{Results}

\section{The location of decidual dendritic cells}

$\mathrm{CD} 3^{+}$DCs were found in decidual samples from both RSA and normal pregnancies. DCs displayed membrane staining, cytoplasmic staining, and appropriate morphological features. Most of the CD83 ${ }^{+} \mathrm{DCs}$ in the decidua from women with RSA were clustered with other DCs (Figure 1A), and few of them were present as single cells in the stroma. The localization of $\mathrm{CD} 83^{+} \mathrm{DCs}$ in the decidua of women with normal pregnancies was similar to that seen for women with RSA. However, most of the decidual $\mathrm{CD} 83^{+} \mathrm{DCs}$ from women with normal pregnancies were present as single cells in the stroma and were not clustered with other DCs (Figure 1B). Very few of $C D 83^{+}$DCs were located close to maternal glandular epithelium. The decidua from all groups were found to contain DCs that stained with the anti-CD1a antibody. $\mathrm{CD}_{1}{ }^{+} \mathrm{DCs}$ displayed membrane staining, cytoplasmic staining, and branch-like morphological features. Most of the decidual $\mathrm{CD} \mathrm{a}^{+} \mathrm{DCs}$ in the two groups were located close to maternal glandular epithelium and uterine epithelium (Figure 2A, B). There was no difference in the location of $\mathrm{CD}_{1}{ }^{+} \mathrm{DCs}$ in the decidua between the two groups.

\section{Dendritic cell counts}

The number of $\mathrm{CD} 83^{+} \mathrm{DCs}$ of decidual tissue from women with RSA was $14.20 \pm 13.34 / 30 \mathrm{HPF}$ and from women with normal pregnancies was $4.77 \pm 2.64 / 30$ HPF. There were significantly more $C D 83^{+} \mathrm{DCs}$ in the decidua of women with RSA than in women with normal pregnancies $(t=3.800, P=0.001)$. The number of decidual $\mathrm{CD} \mathrm{a}^{+}$DCs in RSA and normal pregnancies groups were $3.97 \pm 3.75 / 30 \mathrm{HPF}$ and $7.60 \pm 6.08 / 30 \mathrm{HPF}$, respectively. There were more $\mathrm{CD} 1 \mathrm{a}^{+} \mathrm{DCs}$ in the decidua from normal pregnancies than RSA, and the difference was significant $(t=2.786, P=0.008)$.

\section{Discussion}

The maternal-fetal relationship is a unique immunologic phenomenon. Our research revealed that most of the $\mathrm{mDCs}$ in the decidua from the two groups were located in the stroma. There were more $\mathrm{CD} 83^{+} \mathrm{DCs}$ clustered with other DCs in the stroma from women with RSA than from women with normal pregnancies. CD $83^{+}$DCs' aggregate suggests that these DCs may be immunologically active. Our results were somewhat different from the results of others. Askelund et al. [19] reported that most of the $\mathrm{CD} 83^{+} \mathrm{DCs}$ in the decidua from women with RSA and normal pregnancies were present as single cells in the stroma. It might be that many of the DCs distributed randomly throughout the decidual stroma were also in close proximity to $\mathrm{T}$ cells so they might also be immunologically active. The difference may be due to the different maturation status of DCs and the small number of decidual samples in both studies. Kammerer et al. [20] found 

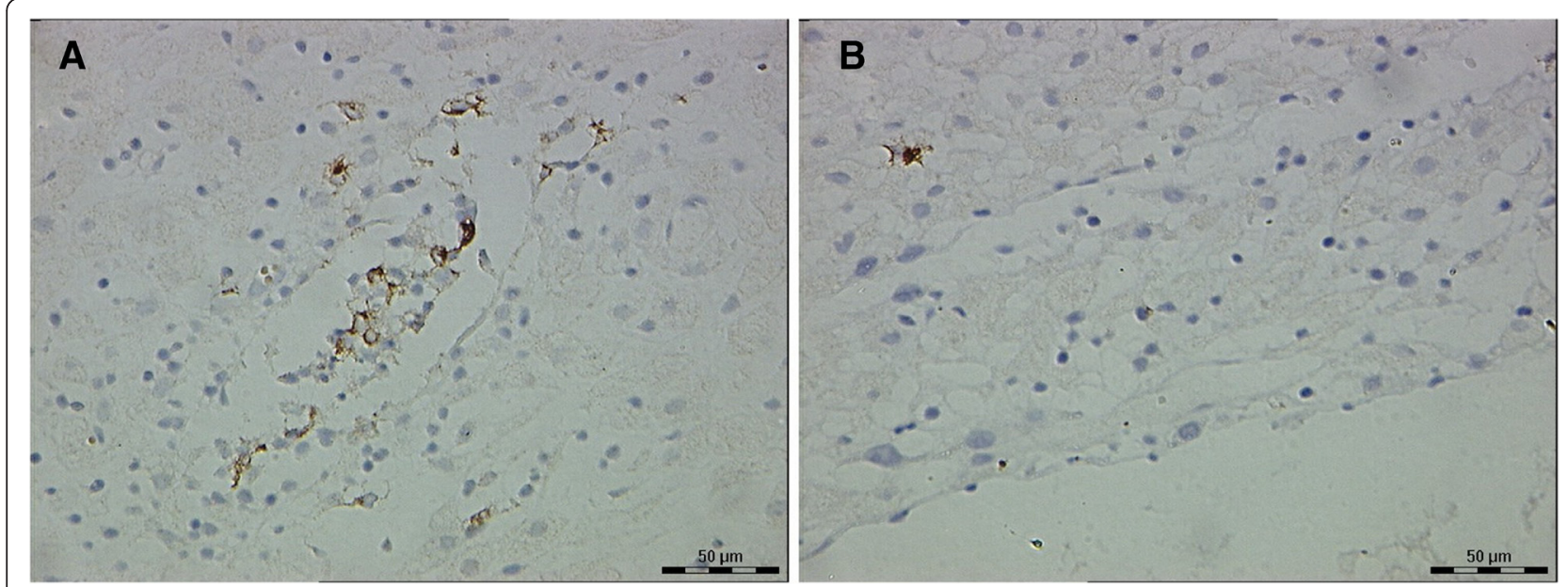

Figure $1 \mathrm{CD}^{+} 3^{+}$dendritic cells (DCs) in the decidua of the two groups. (A) Decidua of recurrent spontaneous abortion (RSA) (a few of CD83 ${ }^{+} \mathrm{DCs}$ clustered in the stroma, $40 \times 10$ ). (B) Decidua of normal pregnancies (a single CD83 ${ }^{+} \mathrm{DC}$ in the stroma, with membrane staining, cytoplasmic staining and branch-like morphology, $40 \times 10$ ).

that $\mathrm{CD} 83^{+}$cells were localized to the basal layer of the decidua in normal early pregnancies. It may be due to our decidual samplings, which were more superficial. It is also possible that the different distribution of DCs throughout the decidua may represent the early stages of a maternal immune response to the failed pregnancy, rather than a cause of pregnancy failure. However, this seems unlikely as the time delay between embryonic demise and curettage was no more than 2 days in our study.

$\mathrm{CD} \mathrm{a}^{+}$DCs were found in both decidual samples of RSA and normal pregnancies. Most of the decidual $\mathrm{CD} 1 \mathrm{a}^{+} \mathrm{DCs}$ in two groups were located close to maternal glandular epithelium or uterine epithelium and there was no difference between two groups. There were some discrepancies between our data and those from Gardner et al. [13], who reported an absence of CD1a expression in decidual leukocytes and no cells in the stroma stained for CD1a at 7 to 12 weeks gestation. The difference between our results and those of Gardner et al. may be due to the different experimental method (immunofluorescence labeling for flow cytometry) used by Gardner et al. We consider that the glandular epithelium and uterine epithelium are the primary position of the local maternal-fetal immune response. Maternal glandular epithelium is close to gestational sac (the maternal-fetal interface). DCs and other immune cells may be involved in gathering the local immune response in the uterine decidua during the embryo's implantation.
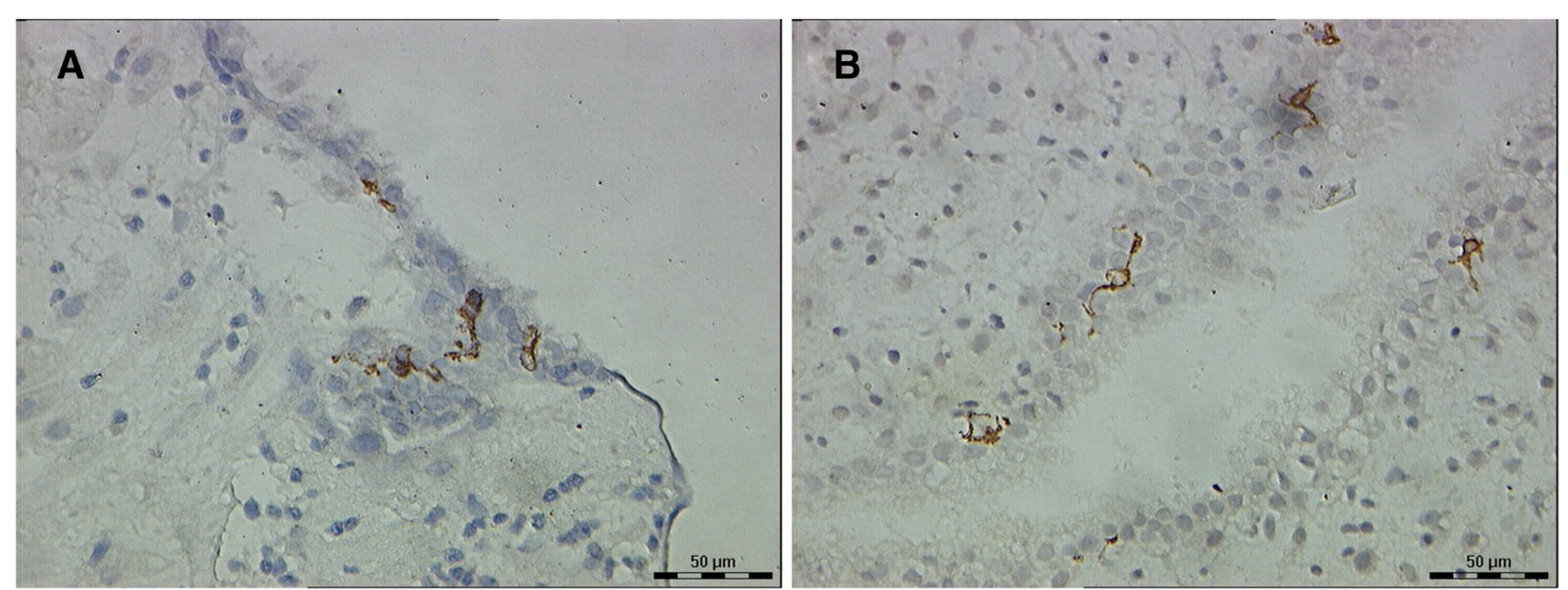

Figure $2 \mathrm{CD} \mathrm{a}^{+}$dendritic cells (DCs) in the decidua of the two groups. (A) Decidua of recurrent spontaneous abortion (RSA) (some CD1a ${ }^{+}$DCs were located close to uterine epithelium, $40 \times 10$ ). (B) Decidua of normal pregnancies (a few of CD1a ${ }^{+}$DCs were located close to glandular epithelium, with membrane staining, cytoplasmic staining and branch-like morphology, $40 \times 10$ ). 
This investigation showed that the mean decidual $\mathrm{CD} 3^{+} \mathrm{DCs}$ population in RSA was higher than the mean population in the normal pregnancies and was statistically significant. This observation suggests the increase in the number of $\mathrm{mDCs}$ probably related to the pathogenesis of RSA. This is different to the findings by Askelund et al. [19]. They did not find significant differences in DCs numbers between the women with recurrent miscarriage and women with normal pregnancies. We assume that an active immune response exists in the RSA decidua. The maturation of DCs determines the outcome of immune response. IDCs can lead to immune tolerance in the physiological condition and require a maturation step that promotes antigen presentation to prime the $\mathrm{T}$ cell activity and trigger the immune response. IDCs transform into $\mathrm{mDCs}$ and induce immune response under the influence of mature-signals (such as bacterial DNA, proinflammatory cytokines, etc.). DCs initiate the function of T cell strongly at this time.

DCs are responsible for stimulating virgin $\mathrm{T}$ cells, a property that distinguishes them from other APC. The action of cytotoxic $\mathrm{T}$ cells in large part determines whether an allograft survives or is rejected. DCs are involved in regulating the balance between $\mathrm{Th} 1$ and Th2, and the Th1/Th2 balance regulates the maintenance of the pregnancy. The maternal balance between Th1 and Th2 type cytokines contributes to the success of the pregnancy [21,22]. Th1-type cytokines (such as IL-2, IL-12, IFN- $\gamma$, TNF- $\beta$, etc.) can defend against microbial infections, and also may lead to abortion. For example, IL-12 can promote a T cell to Th1-type biased, induced immune response. However, Th2-type cytokines (such as IL-4, IL-5, IL-10, etc.) can lead to less autoimmunity, susceptible to infections, and also may induce pregnancytolerance to fetus, providing protection to pregnancy. For instance, IL-10 can induce T-cell regulation (regulatory T cells, Treg) and promote monocyte-derived DCs induce antigen-specific $\mathrm{CD} 4^{+}$and $\mathrm{CD} 8^{+} \mathrm{T}$ cell immune incompetence [23], which can inhibit immune response of T-cell and induce tolerance of T cell [24].

Research on mouse models [25] showed that fetal antigens on the placenta during the implant period could stimulate DCs in the decidua to produce Th1-type cytokines. Th2-type cytokines then dominated. The level of IL-10 in placenta gradually increased with the development of pregnancy in normal pregnancy. High concentrations of IL-10 may inhibit the decidual DCs' ability to produce IL-12 and then regulates the Th1/Th2 balance to Th2 and shift the direction of the end-mediated immune tolerance to the fetus. Decidual iDCs uptake antigens and then transform into $\mathrm{mDCs}$ with antigen presenting function, which can induce and stimulate the proliferation of naive $\mathrm{T}$ cells. The maternal immune response breaks the balance between Th1/Th2, and makes the maternal immune response to Th1-type. The above reaction leads to a semi-allograft immune response to the fetal and placenta by mother and occurrence of miscarriage eventually.

IDCs are characterized by the CD1a expression. We found lesser $C D 1 a^{+}$DCs in the decidua of RSA than in the decidua from normal pregnancies. This suggests that the decrease in the quantity of iDCs is probably related to the occurrence of RSA. It is possible that decidual iDCs play a role in the maintenance of normal pregnancy through the regulation of maternal-fetal immune tolerance [26]. Most of the DCs in the nonlymphoid organs are immature. Studies found that some DCs subtypes in human decidua may play an important role to maintain pregnancy $[27,28]$. Decidual DCs may have a significant protective effect in miscarriage in pregnant mice [2]. Therapy with DCs can reduce the spontaneous abortion rate, through a mechanism whereby DCs therapy differentially upregulated a regulatory/protective population of cells at the fetal-maternal interface [18].

DCs may regulate the Treg cells to suppress the semiallograft rejection from mother to the fetus and play an important role in the maternal-fetal immune tolerance. Treg cells have significant roles in the negative regulation of immune response and induction of immune tolerance. $\mathrm{CD} 4{ }^{+} \mathrm{CD} 25^{+}$Treg cells exist in both maternal blood and decidua throughout pregnancy. Zenclussen et al. [29] reported Treg cells were proposed to play an essential role. Normal pregnant mice show an expansion of $\mathrm{CD} 4^{+} \mathrm{CD} 25^{+}$and IL- $10^{+}$Treg cells at the periphery compared to nonpregnant animals. Further, they suggested significantly lower frequencies of Treg cells in abortion-prone mice. $\mathrm{CD} 4^{+} \mathrm{CD} 25^{+}$Treg cells from normal pregnant mice were able to prevent fetal rejection. Accordingly, downregulated levels of Treg cells were also reported during human miscarriage. There was an increase in circulating Treg cells during early pregnancy, peaking during the second-trimester [30]. In peripheral blood, statistically significantly higher proportions of $\mathrm{CD} 4{ }^{+} \mathrm{CD} 25^{+} \mathrm{T}$ cells expression was observed in normal early pregnant women compared with normal nonpregnant women and unexplained RSA patients. There were statistically significantly lower proportions of $\mathrm{CD} 4^{+} \mathrm{CD} 25^{+} \mathrm{T}$ cells expression in unexplained RSA patients compared with normal early pregnant women in their decidua [31].

IDCs inhibit proliferation of antigen-specific $\mathrm{T}$ cell by self-secretion of IL-10. IDCs induce $\mathrm{CD} 44^{+} \mathrm{CD} 25^{+}$Treg and $\mathrm{CD} 8^{+}$suppressor $\mathrm{T}$ cells, which inhibit the immune response [32]. IL-10 secreted by DCs was increased when there is interaction between DCs and Treg cells. DCs and Treg cells formed a feedback inhibition [33]. IDCs in the peripheral tissue induce removal of antigenspecific $T$ cells and induce peripheral tolerance under normal physiological conditions without infection and 
tissue damage. The immune system identifies and defends 'danger signals' from tissue damage to maintain tolerance of self-antigens and harmless exogenous antigens through this mechanism.

\section{Conclusions}

In spite of an increasing interest, the etiology of RSA remains unknown in approximately 50 percent of cases. This study demonstrates significant associations between the maturation of decidual DCs and RSA. The increase in the number of $\mathrm{mDCs}$ and the decrease in the number of iDCs may be related to RSA. As mDCs are important antigen presenters, these observations suggest the existence of an active immune response in the decidua of women with RSA. DCs play an important role in the aetiology of RSA. However, its specific pathogenesis has not been clarified exactly yet, and the immunologic nature of RSA has generated considerable interest and controversy. Many factors could be correlated to RSA. For example, passive smoking, a higher body mass index (BMI), and a family history of RSA were thought to be independent risk factors for RSA [34]. Further researches are needed to characterize the specific functions of decidual DCs. Despite awareness of the limitations of this study due to the small sample size and semi-quantitative evaluation, the results encourage further larger studies in this promising research field.

\section{Consent}

Written informed consent was obtained from each of the patients for publication of this report and any accompanying images. A copy of this written consent is available from each patient for review by the Editor-in-Chief of this journal.

\footnotetext{
Abbreviations

APC: antigen-presenting cells; BMI: body mass index; DCs: dendritic cells; HLA: human leukocyte antigen; HPF: high-power fields; iDCs: immature DCs; mDCs: mature DCs; NK cells: natural killer cells; RSA: recurrent spontaneous abortion; Th1: T-helper-1 type response; Th2: T-helper-2 type response; Treg: regulatory $T$ cells.
}

\section{Competing interests}

The authors declare that they have no competing interests.

\section{Authors' contributions}

ZDQ, LLH, and XMZ designed the study, collected the patients' information, completed the experiments, drafted the manuscript, and performed the statistical analysis. All authors read and approved the final manuscript.

\section{Acknowledgements}

This work was supported by the General Research Program of Education Department of Zhejiang Province, P. R. China (No.:Y200804440) , Program for Zhejiang Leading Team of S \& T Innovation, P. R. China (No.: 2011R50013-25), Program for Key Subjects of Zhejiang Province in Medicine \& Hygiene, P. R. China (No.: 11-ZC18), Program for Key Subjects Group of Reproductive Medicine at Zhejiang Province, P. R. China (No.: XKQ-009-002), Program for Changjiang Scholars and Innovative Research Team in University, P. R. China (No: IRT1184), and the National Basic Research Program of China (2012CB944902).
Received: 20 January 2014 Accepted: 8 December 2014

Published online: 07 January 2015

\section{References}

1. Rai R, Regan L: Recurrent miscarriage. Lancet 2006, 368:601-611.

2. Blois S, Alba Soto CD, Olmos S, Chuluyan E, Gentile T, Arck PC, Margni RA: Therapy with dendritic cells influences the spontaneous resorption rate in the CBA/J x DBA/2 J mouse model. Am J Reprod Immunol 2004, 51:40-48.

3. Kammerer U: Antigen-presenting cells in the decidua. Chem Immunol Allerg 2005, 89:96-104.

4. Sanguansermsri D, Pongcharoen S: Pregnancy immunology: decidual immune cells. Asian Pac J Allergy Immunol 2008, 26:171-181.

5. Steinman RM: The dendritic cell system and its role in immunogenicity. Annu Rev Immunol 1991, 9:271-296.

6. Banchereau J, Steinman RM: Dendritic cells and the control of immunity. Nature 1998, 392:245-252.

7. Banchereau J, Briere F, Caux C, Davoust J, Lebecque S, Liu YJ, Pulendran B, Palucka K: Immunobiology of dendritic cells. Annu Rev Immunol 2000, 18:767-811.

8. Chorny A, Gonzalez-Rey E, Delgado M: Regulation of dendritic cell differentiation by vasoactive intestinal peptide: therapeutic applications on autoimmunity and transplantation. Ann NY Acad Sci 2006, 1088:187-194.

9. Reid DC: Dendritic cells and immunotherapy for malignant cells. $\mathrm{Br} J$ Haematol 2001, 112:874-875.

10. Abraham S, Indrasingh I, Vettivel S, Chandi G: Gross morphology and ultrastructure of dendritic cells in the normal human decidua. Clin Anat 2000, 13:177-180.

11. Prechtel AT, Steinkasserer A: CD83: an update on functions and prospects of the maturation marker of dendritic cells. Arch Dermatol Res 2007, 299:59-69.

12. Coventry BJ, Austyn JM, Chryssidis S, Hankins D, Harris A: Identification and isolation of CD1a positive putative tumour infiltrating dendritic cells in human breast cancer. Adv Exp Med Biol 1997, 417:571-577.

13. Gardner L, Moffett A: Dendritic cells in the human decidua. Biol Reprod 2003, 69:1438-1446.

14. von Bergwelt-Baildon MS, Popov A, Saric T, Chemnitz J, Classen S, Stoffel MS, Fiore F, Roth U, Beyer M, Debey S, Wickenhauser C, Hanisch FG, Schultze JL: CD25 and indoleamine 2,3-dioxygenase are up-regulated by prostaglandin E2 and expressed by tumor-associated dendritic cells in vivo: additional mechanisms of T-cell inhibition. Blood 2006, 108:228-237.

15. Koya T, Matsuda H, Takeda K, Matsubara S, Miyahara N, Balhorn A, Dakhama A, Gelfand EW: IL-10-treated dendritic cells decrease airway hyperresponsiveness and airway inflammation in mice. J Allergy Clin Immunol 2007, 119:1241-1250.

16. Khanna A, Morelli AE, Zhong C, Takayama T, Lu L, Thomson AW: Effects of liver-derived dendritic cell progenitors on Th1-and Th2-like cytokine responses in vitro and in vivo. J Immunol 2000, 164:1346-1354.

17. Blois SM, Barrientos G, Garcia MG, Orsal AS, Tometten M, Cordo-Russo RI, Klapp BF, Santoni A, Fernández N, Terness P, Arck PC: Interaction between dendritic cells and natural killer cells during pregnancy in mice. $J \mathrm{Mol}$ Med 2008, 86:837-852.

18. Miranda S, Litwin S, Barrientos G, Szereday L, Chuluyan E, Bartho JS, Arck PC, Blois SM: Dendritic cells therapy confers a protective microenvironment in murine pregnancy. Scand J Immunol 2006, 64:493-499.

19. Askelund K, Liddell HS, Zanderigo AM, Fernando NS, Khong TY, Stone PR, Chamley LW: CD83(+) dendritic cells in the decidua of women with recurrent miscarriage and normal pregnancy. Placenta 2004, 25:140-145.

20. Kämmerer U, Schoppet M, McLellan AD, Kapp M, Huppertz HI, Kämpgen $E$, Dietl J: Human decidua contains potent immunostimulatory CD83(+) dendritic cells. Am J Pathol 2000, 157:159-169.

21. Dealtry GB, O'Farrell MK, Fernandez N: The Th2 cytokine environment of the placenta. Int Arch Allergy Immunol 2000, 123:107-119.

22. Chaouat G, Ledee-Bataille N, Dubanchet S, Zourbas S, Sandra O, Martal J: $\mathrm{TH} 1 / \mathrm{TH} 2$ paradigm in pregnancy: paradigm lost? Cytokines in pregnancy/ early abortion: reexamining the $\mathrm{TH} 1 / \mathrm{TH} 2$ paradigm. Int Arch Allergy Immunol 2004, 134:93-119.

23. Cools N, Van Tendeloo VF, Smits EL, Lenjou M, Nijs G, Van Bockstaele DR Berneman ZN, Ponsaerts P: Immuno-suppression induced by immature dendritic cells is mediated by TGF-beta/IL-10 double - positive CD4 + regulatory T cells. J Cell Mol Med 2008, 12:690-700. 
24. Encabo A, Solves P, Carbonell-Uberos F, Minana MD: The functional immaturity of dendritic cells can be relevant to increased tolerance associated with cord blood transplantation. Transfusion 2007, 47:272-279.

25. Blois SM, Alba Soto CD, Tometten M, Klapp BF, Margni RA, Arck PC: Lineage, maturity, and phenotype of uterine murine dendritic cells throughout gestation indicate a protective role in maintaining pregnancy. Biol Reprod 2004, 70:1018-1023.

26. Miwa N, Hayakawa S, Miyazaki S, Myojo S, Sasaki Y, Sakai M, Takikawa O, Saito S: IDO expression on decidual and peripheral blood dendritic cells and monocytes/macrophages after treatment with CTLA-4 or interferon- $\gamma$ increase in normal pregnancy but decrease in spontaneous abortion. Mol Hum Reprod 2005, 11:865-870.

27. Ban YL, Kong BH, Qu X, Yang QF, Ma YY: BDCA-1+, BDCA-2+ and BDCA-3+ dendritic cells in early human pregnancy decidua. Clin Exp Immunol 2008, 151:399-406.

28. Kammerer U, Kruse A, Barrientos G, Arck PC, Blois SM: Role of dendritic cells in the regulation of maternal immune responses to the fetus during mammalian gestation. Immunol Invest 2008, 37:499-533.

29. Zenclussen $A C: C D 4+C D 25+$ regulatory $T$ cells in murine pregnancy. J Reprod Immunol 2005, 65:101-110.

30. Somerset DA, Zheng Y, Kilby MD, Sansom DM, Drayson MT: Normal human pregnancy is associated with an elevation in the immune suppressive CD25 + CD4+ regulatory T-cell subset. Immunology 2004, 112:38-43.

31. Mei S, Tan J, Chen H, Chen Y, Zhang J: Changes of CD4 + CD25 high regulatory $\mathrm{T}$ cells and FOXP3 expression in unexplained recurrent spontaneous abortion patients. Fertil Steril 2010, 94:2244-2247.

32. Savina A, Jancic C, Hugues $S$, Guermonprez P, Vargas P, Moura IC, Lennon-Duménil AM, Seabra MC, Raposo G, Amigorena S: NOX2 controls phagosomal $\mathrm{PH}$ to regulate antigen processing during crosspresentation by dendritic cells. Cell 2006, 126:205-218.

33. Misra N, Bayry J, Lacroix-Desmazes S, Kazatchkine MD, Kaveri SV: Cutting edge human $\mathrm{CD} 4+\mathrm{CD} 25+\mathrm{T}$ cells restrain the maturation and antigen-presenting function of dendritic cells. J Immunol 2004, 172:4676-4680.

34. Zhang BY, Wei YS, Niu JM, Li Y, Miao ZL, Wang ZN: Risk factors for unexplained recurrent spontaneous abortion in a population from southern China. Int J Gynaecol Obstet 2010, 108:135-138.

\section{Submit your next manuscript to BioMed Central and take full advantage of:}

- Convenient online submission

- Thorough peer review

- No space constraints or color figure charges

- Immediate publication on acceptance

- Inclusion in PubMed, CAS, Scopus and Google Scholar

- Research which is freely available for redistribution 